\title{
A Two-Dimensional Spectrum for Bistatic SAR Processing Using Series Reversion
}

\author{
Yew Lam Neo, Student Member, IEEE, Frank Wong, and Ian G. Cumming, Life Senior Member, IEEE
}

\begin{abstract}
This letter derives the two-dimensional point target spectrum for an arbitrary bistatic synthetic aperture radar configuration. The method described makes use of series reversion, the method of stationary phase, and Fourier transform pairs to derive the point target spectrum. The accuracy of the spectrum is controlled by keeping enough terms in the two series expansions, and is verified with a point target simulation.
\end{abstract}

Index Terms-Bistatic SAR, point target spectrum, SAR simulation, series reversion, synthetic aperture radar (SAR).

\section{INTRODUCTION}

$\mathbf{T}$ HE IDEAL solution for bistatic synthetic aperture radar (SAR) image formation is a two-dimensional (2-D) matched filtering process. The time-domain method [1] is a direct matched filtering of the baseband signal using the exact replica of the echo signal at each location and thus gives the optimum reconstruction. However, this method is computationally intensive as it scales with an order of $O\left((N \times M)^{2}\right)$, where $N \times M$ is the number of pixels in the image.

Efficiency can be improved by performing the focusing in the frequency domain. The point target spectrum is the basis for most efficient processing algorithms operating in the 2-D or range Doppler domain [2]. The individual transmitter and receiver range histories are hyperbolic, as in the monostatic case. However, because the transmit and receive range equations are not the same in the bistatic case, the total range is no longer a hyperbola. This means that the point target spectrum of the monostatic and bistatic cases are inherently different and thus, in general, monostatic algorithms are not able to focus bistatic configurations.

The point target spectrum for the monostatic case has been derived in [3], and an approximate point target spectrum for some bistatic cases has been derived in [4]. In [5], it was shown how the 2-D spectrum can be modified to change the leader-follower bistatic case with a constant baseline into an equivalent monostatic case for which a conventional monostatic algorithm can be applied. In this letter, the 2-D frequency spectrum is derived for the general bistatic case, based on the reversion of a series approximation. A simulation is performed to illustrate its accuracy.

Manuscript received June 1, 2006; revised July 10, 2006.

Y. L. Neo is with the DSO National Laboratories, Singapore 118230.

F. Wong is with MacDonald, Dettwiler and Associates Ltd., Richmond, BC V6V 2J3, Canada.

I. G. Cumming is with the Department of Electrical and Computer Engineering, University of British Columbia, Vancouver, BC V6T 1Z4, Canada.

Digital Object Identifier 10.1109/LGRS.2006.885862

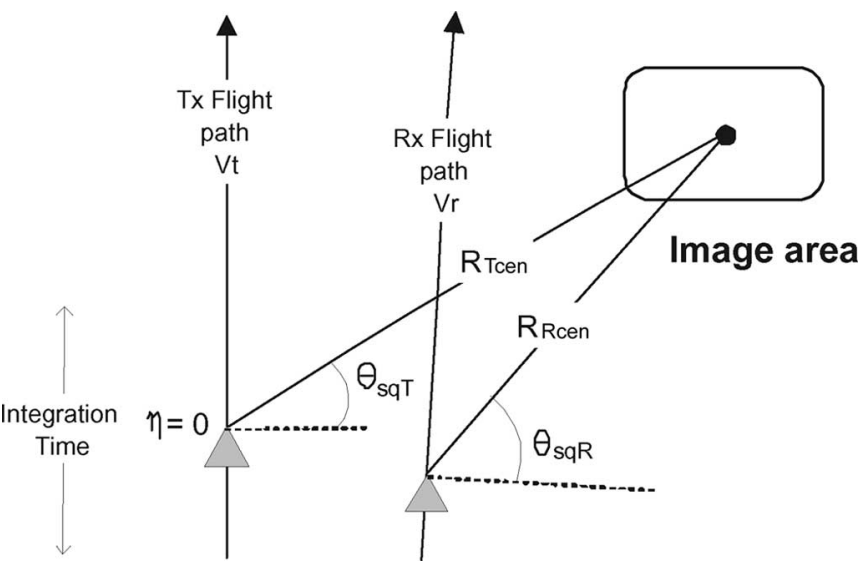

Fig. 1. General bistatic configuration of transmitter and receiver at $\eta=0$.

The results of this letter will be useful for developing efficient bistatic algorithms operating in the 2-D frequency domain or the range Doppler domain. Several bistatic airborne experiments involving geometries with fixed baseline [6] and [7] were conducted recently. For these flight configurations, point targets with the same closest range of approach would have the same range Doppler histories. Thus, using the same point target spectrum, we are able to focus a family of points and hence achieve processing efficiency. In another paper [8], this method was used to develop an efficient frequency-domain matched filter.

\section{Bistatic SAR Signal Model}

A general bistatic SAR geometry is shown in Fig. 1, including nonparallel tracks, unequal velocities, and antenna squint. The time-domain matched filter is constructed by forming the instantaneous slant range to a point target, referred to as the range equation

$$
\begin{aligned}
R(\eta)= & R_{\mathrm{T}}(\eta)+R_{\mathrm{R}}(\eta) \\
= & \sqrt{V_{\mathrm{T}}^{2} \eta^{2}+R_{\text {Tcen }}^{2}-2 V_{\mathrm{T}} \eta R_{\mathrm{Tcen}} \sin \theta_{\mathrm{sqT}}} \\
& +\sqrt{V_{\mathrm{R}}^{2} \eta^{2}+R_{\mathrm{Rcen}}^{2}-2 V_{\mathrm{R}} \eta R_{\mathrm{Rcen}} \sin \theta_{\mathrm{sqR}}}
\end{aligned}
$$

where $\eta$ is azimuth time, $V$ is the velocity of the platform, $R$ is the instantaneous range to the point target, and the subscripts $\mathrm{T}$ and $\mathrm{R}$ refer to the transmitter and receiver, respectively. The subscript, cen, refers to a target at the center of the imaged area.

Zero azimuth time $(\eta=0)$ is defined as the midpoint of the integration path for the transmitter, as shown in Fig. 1. The receiver position is shown at the same time. $\theta_{\mathrm{sqT}}$ is the squint 
angle of the transmitter, and $\theta_{\mathrm{sqR}}$ is the squint angle of the receiver at this time.

After demodulation to baseband, the received signal can be written in terms of the range time (fast time) $\tau$ and azimuth time (slow time) $\eta$

$$
s(\tau, \eta)=\rho_{r}\left(\tau-\frac{R(\eta)}{c}\right) w_{\mathrm{az}}(\eta) \exp \left\{-j 2 \pi \frac{R(\eta)}{\lambda}\right\}
$$

where $\rho_{r}(\cdot)$ is the range envelope and the azimuth envelope $w_{\mathrm{az}}(\cdot)$ is determined by the composite antenna pattern.

\section{Derivation of the Signal Spectrum}

To derive the 2-D spectrum, the first step is to remove the linear phase and the linear range cell migration (LRCM). This reason for this step will become apparent when we apply the series reversion at a later step. After removal of these terms, the point target signal in the time domain is

$s_{A}(\tau, \eta)=\rho_{r}\left(\tau-\frac{R_{1}(\eta)}{c}\right) w_{\text {az }}(\eta) \exp \left\{-j 2 \pi \frac{R_{1}(\eta)}{\lambda}\right\}$

where

$$
R_{1}(\eta)=R_{\text {cen }}+k_{2} \eta^{2}+k_{3} \eta^{3}+k_{4} \eta^{4}+\cdots
$$

is the range after removing the linear term and $R_{\text {cen }}$ is the sum of $R_{\text {Tcen }}$ and $R_{\text {Rcen }}$, and the coefficients

$$
\begin{aligned}
k_{2} & =\left.\frac{1}{2 !}\left(\frac{d R_{\mathrm{T}}^{2}(\eta)}{d \eta^{2}}+\frac{d R_{\mathrm{R}}^{2}(\eta)}{d \eta^{2}}\right)\right|_{\eta=0} \\
k_{3} & =\left.\frac{1}{3 !}\left(\frac{d R_{\mathrm{T}}^{3}(\eta)}{d \eta^{3}}+\frac{d R_{\mathrm{R}}^{3}(\eta)}{d \eta^{3}}\right)\right|_{\eta=0} \\
k_{4} & =\left.\frac{1}{4 !}\left(\frac{d R_{\mathrm{T}}^{4}(\eta)}{d \eta^{4}}+\frac{d R_{\mathrm{R}}^{4}(\eta)}{d \eta^{4}}\right)\right|_{\eta=0} \\
\ldots &
\end{aligned}
$$

are evaluated at the aperture center. The derivatives of the transmitter range are given by

$$
\begin{aligned}
& \left.\frac{d^{2} R_{\mathrm{T}}(\eta)}{d \eta^{2}}\right|_{\eta=0}=\frac{V_{\mathrm{T}}^{2} \cos ^{2} \theta_{\mathrm{sqT}}}{R_{\mathrm{Tcen}}} \\
& \left.\frac{d^{3} R_{\mathrm{T}}(\eta)}{d \eta^{3}}\right|_{\eta=0}=\frac{3 V_{\mathrm{T}}^{3} \cos ^{2} \theta_{\mathrm{sqT}} \sin \theta_{\mathrm{sqT}}}{R_{\mathrm{Tcen}}^{2}} \\
& \left.\frac{d^{4} R_{\mathrm{T}}(\eta)}{d \eta^{4}}\right|_{\eta=0}=\frac{3 V_{\mathrm{T}}^{4} \cos ^{2} \theta_{\mathrm{sqT}}\left(4 \sin ^{2} \theta_{\mathrm{sqT}}-\cos ^{2} \theta_{\mathrm{sqT}}\right)}{R_{\mathrm{Tcen}}^{3}} .
\end{aligned}
$$

Similar equations can be written for the derivatives of the receiver range $R_{\mathrm{R}}(\eta)$.

If we keep the terms up to the fourth-order term in (8) and expand up to the fourth azimuth frequency term, the 2-D point target spectrum is given by

$$
S_{A}^{\prime}\left(f_{\tau}, \eta\right)=W_{\mathrm{r}}\left(f_{\tau}\right) w_{\mathrm{az}}(\eta) \exp \left\{-j 2 \pi \frac{\left(f_{\mathrm{o}}+f_{\tau}\right) R_{1}(\eta)}{c}\right\}
$$

where $W_{\mathrm{r}}(\cdot)$ represents the spectral shape (e.g., bandwidth) of the transmitted pulse, $f_{\mathrm{o}}$ corresponds to the center frequency, and $f_{\tau}$ is the range frequency. Next, we perform an azimuth Fourier transform (FT). Using the method of stationary phase [9], azimuth frequency is related to azimuth time by

$$
\left(-\frac{c}{f_{\mathrm{o}}+f_{\tau}}\right) f_{\eta}=2 k_{2} \eta+3 k_{3} \eta^{2}+4 k_{4} \eta^{3}+\cdots
$$

where $f_{\eta}$ is the azimuth frequency. We can derive an expression of $\eta$ in terms of $f_{\eta}$ by using the series reversion (refer to the Appendix). In the forward function (26), we replace $x$ by $\eta, y$ by $\left(-c /\left(f_{\mathrm{o}}+f_{\tau}\right)\right) f_{\eta}$, and substitute the coefficients of $x$ by the coefficients of $\eta$. Inverting this power series, we arrive at

$$
\begin{aligned}
\eta\left(f_{\eta}\right)=A_{1}\left(-\frac{c}{f_{\mathrm{o}}+f_{\tau}} f_{\eta}\right) & +A_{2}\left(-\frac{c}{f_{\mathrm{o}}+f_{\tau}} f_{\eta}\right)^{2} \\
& +A_{3}\left(-\frac{c}{f_{\mathrm{o}}+f_{\tau}} f_{\eta}\right)^{3}+\cdots
\end{aligned}
$$

The rationale for removal of the linear phase term and LRCM becomes clear at this step. In order to apply the series reversion directly in (12), we should remove the constant term in the forward function since the constant term is absent in the forward function (26). Both the linear phase term and the LRCM term are removed so that there is no constant term left after applying azimuth FT to (11). ${ }^{1}$

Using (13) with (11), we obtain the 2-D spectrum of $s_{A}(\tau, \eta)$

$$
\begin{aligned}
S_{A}\left(f_{\tau}, f_{\eta}\right)=W_{\mathrm{r}} & \left(f_{\tau}\right) W_{\mathrm{az}}\left(f_{\eta}\right) \exp \left\{-j 2 \pi f_{\eta} \eta\left(f_{\eta}\right)\right\} \\
& \times \exp \left\{-j \frac{2 \pi\left(f_{\mathrm{o}}+f_{\tau}\right)}{c} R_{1}\left(\eta\left(f_{\eta}\right)\right)\right\}
\end{aligned}
$$

where $W_{\mathrm{az}}(\cdot)$ represents the shape of the Doppler spectrum and is approximately a scaled version of the azimuth time envelope $w_{\mathrm{az}}(\cdot)$. To get the 2-D point target spectrum for $s(\tau, \eta)$, we reintroduce the LRCM and linear phase into $s_{A}(\tau, \eta)$ in (3)

$$
\begin{aligned}
s(\tau, \eta)= & s_{A}\left(\tau-\frac{k_{1} \eta}{c}, \eta\right) \exp \left\{-j 2 \pi \frac{f_{\mathrm{o}} k_{1}}{c} \eta\right\} \\
= & \rho_{r}\left(\tau-\frac{R_{1}(\eta)+k_{1} \eta}{c}\right) w_{\mathrm{az}}(\eta) \\
& \times \exp \left\{-j 2 \pi\left(\frac{f_{\mathrm{o}} R_{1}(\eta)}{c}+\frac{f_{\mathrm{o}} k_{1} \eta}{c}\right)\right\}
\end{aligned}
$$

where

$$
k_{1}=\left.\frac{d R_{\mathrm{T}}(\eta)}{d \eta}\right|_{\eta=0}+\left.\frac{d R_{\mathrm{R}}(\eta)}{d \eta}\right|_{\eta=0} .
$$

The derivatives (16) at the aperture center are given by

$$
\begin{aligned}
& \left.\frac{d R_{\mathrm{T}}(\eta)}{d \eta}\right|_{\eta=0}=-V_{\mathrm{T}} \sin \theta_{\mathrm{sqT}} \\
& \left.\frac{d R_{\mathrm{R}}(\eta)}{d \eta}\right|_{\eta=0}=-V_{\mathrm{R}} \sin \theta_{\mathrm{sqR}} .
\end{aligned}
$$

\footnotetext{
${ }^{1}$ An alternate approach is to move the constant term to the left-hand side of (12) and treat the whole term on the left-hand side as $y$. We would still end up with the same solution (20).
} 
TABLE I

SimUlation PARAMETERS

\begin{tabular}{|c|c|c|}
\hline Simulation parameters & Transmitter & Receiver \\
\hline \hline Velocity in x direction & $0 \mathrm{~m} / \mathrm{sec}$ & $20 \mathrm{~m} / \mathrm{sec}$ \\
\hline Velocity in y direction & $180 \mathrm{~m} / \mathrm{sec}$ & $220 \mathrm{~m} / \mathrm{sec}$ \\
\hline Velocity in z direction & $0 \mathrm{~m} / \mathrm{sec}$ & $0 \mathrm{~m} / \mathrm{sec}$ \\
\hline Center frequency & \multicolumn{2}{|c|}{$5.00 \mathrm{GHz}$} \\
\hline Range bandwidth & \multicolumn{2}{|c|}{$50 \mathrm{MHz}$} \\
\hline Doppler bandwidth & \multicolumn{2}{|c|}{$150 \mathrm{~Hz}$} \\
\hline Altitude & $3000 \mathrm{~m}$ & $1000 \mathrm{~m}$ \\
\hline Range to point target at $\eta=0$ & $16532 \mathrm{~m}$ & $10444 \mathrm{~m}$ \\
\hline Squint angle at $\eta=0$ & $30^{\circ}$ & $60.2^{\circ}$ \\
\hline Distance between airplanes at $\eta=0$ & \multicolumn{2}{|c|}{$8351 \mathrm{~m}$} \\
\hline Minimum distance between airplanes & \multicolumn{2}{|c|}{$8445 \mathrm{~m}$} \\
\hline Maximum Distance between airplanes & \multicolumn{2}{|c|}{$8261 \mathrm{~m}$} \\
\hline
\end{tabular}

To derive the 2-D point target spectrum for $s(\tau, \eta)$, we use the FT skew and shift properties [2]

$$
\begin{aligned}
g(\tau, \eta) & \longleftrightarrow G\left(f_{\tau}, f_{\eta}\right) \\
g(\tau, \eta) \exp \left\{-j 2 \pi f_{\kappa} \eta\right\} & \longleftrightarrow G\left(f_{\tau}, f_{\eta}+f_{\kappa}\right) \\
g(\tau-\kappa \eta, \eta) & \longleftrightarrow G\left(f_{\tau}, f_{\eta}+\kappa f_{\tau}\right)
\end{aligned}
$$

where $g$ is a 2-D time function, $G$ is its corresponding frequency function, and $\kappa$ and $f_{\kappa}$ are constants. Applying these FT pairs to (14) and (15), we arrive at the desired 2-D point target spectrum

$$
S\left(f_{\tau}, f_{\eta}\right)=S_{A}\left[f_{\tau}, f_{\eta}+\left(f_{\mathrm{o}}+f_{\tau}\right) \frac{k_{1}}{c}\right]
$$

The accuracy of the spectrum is limited by the number of terms used in the expansion of (20). In general, we would like to limit the uncompensated phase error to be within $\pm \pi / 4$, in order to avoid significant deterioration of the image quality.

\section{Simulation Results}

To prove the validity of the formulation, a point target signal is simulated in the time domain and matched filtering is carried out in the 2-D frequency domain. Processing efficiency is achieved by focusing point targets in an invariance region with the same matched filter. The size of the invariance region is dependent upon the radar parameters and the imaging geometry. The purpose of this letter is to prove accuracy of the derived spectrum. Analysis of the extent of the invariance region will be investigated in a separate paper.

The simulation uses airborne SAR parameters given in Table I. An appreciable amount of antenna squint is assumed, as well as unequal platform velocities and nonparallel tracks. The axes are defined in a right-hand Cartesian coordinate system with the flight direction of the transmitter parallel to the $y$ direction and $z$ is the altitude of the aircraft. The oversampling ratio is 1.33 in range and azimuth. Rectangular weighting is used for both azimuth and range processing.
If we keep the terms up to a fourth-order term in (20) and expand up to the fourth azimuth frequency term, the 2-D point target spectrum is given by

$$
S\left(f_{\tau}, f_{\eta}\right)=W_{\mathrm{r}}\left(f_{\tau}\right) W_{\mathrm{az}}\left(f_{\eta}+\left(f_{\mathrm{o}}+f_{\tau}\right) \frac{k_{1}}{c}\right) \exp \left\{j \phi\left(f_{\tau}, f_{\eta}\right)\right\}
$$

where the phase is given by

$$
\begin{aligned}
\phi\left(f_{\tau}, f_{\eta}\right)= & -2 \pi\left(\frac{f_{\mathrm{o}}+f_{\tau}}{c}\right) R_{\text {cen }} \\
& +2 \pi \frac{c}{4 k_{2}\left(f_{\mathrm{o}}+f_{\tau}\right)}\left(f_{\eta}+\left(f_{\mathrm{o}}+f_{\tau}\right) \frac{k_{1}}{c}\right)^{2} \\
& +2 \pi \frac{c^{2} k_{3}}{8 k_{2}^{3}\left(f_{\mathrm{o}}+f_{\tau}\right)^{2}}\left(f_{\eta}+\left(f_{\mathrm{o}}+f_{\tau}\right) \frac{k_{1}}{c}\right)^{3} \\
& +2 \pi \frac{c^{3}\left(9 k_{3}^{2}-4 k_{2} k_{4}\right)}{64 k_{2}^{5}\left(f_{\mathrm{o}}+f_{\tau}\right)^{3}}\left(f_{\eta}+\left(f_{\mathrm{o}}+f_{\tau}\right) \frac{k_{1}}{c}\right)^{4} .
\end{aligned}
$$

The magnitudes of the cubic and quartic terms in (22) are

$$
\begin{aligned}
& \Delta \phi_{3} \approx\left|2 \pi \frac{c^{2} k_{3}}{8 k_{2}^{3} f_{\mathrm{o}}^{2}}\left(\frac{B_{\mathrm{a}}}{2}\right)^{3}\right| \\
& \Delta \phi_{4} \approx\left|2 \pi \frac{c^{3}\left(9 k_{3}^{2}-4 k_{2} k_{4}\right)}{64 k_{2}^{5} f_{\mathrm{o}}^{3}}\left(\frac{B_{\mathrm{a}}}{2}\right)^{4}\right|
\end{aligned}
$$

where $B_{\mathrm{a}}$ is the Doppler bandwidth. For this simulation case, $B_{\mathrm{a}}=150 \mathrm{~Hz}, k_{2}=1.31 \mathrm{~m} / \mathrm{s}, k_{3}=0.0146 \mathrm{~m} / \mathrm{s}^{2}$, and $k_{4}=$ $0.000184 \mathrm{~m} / \mathrm{s}^{3}$. The phase component $\Delta \phi_{3}$ is more than $\pi / 4$ and $\Delta \phi_{4}$ is much less than $\pi / 4$. Therefore, it is sufficient to retain only terms up to the cubic term in the phase expansion (22) for accurate focusing in this radar case. Matched filtering is performed by multiplying the 2-D spectrum of the point target by $\exp \left(-j \phi\left(f_{\tau}, f_{\eta}\right)\right)$.

The point target spectrum after matched filtering has a 2-D envelope given by $W_{\mathrm{r}}$ and $W_{\mathrm{az}}$ in (21), as shown in Fig. 2(a). Note that the spectrum has a skew as a result of the range/azimuth coupling. This results in skewed sidelobes as shown in Fig. 2(b). However, in order to measure image quality parameters such as the $3-\mathrm{dB}$ impulse response width (IRW) and the peak sidelobe ratio (PSLR), it is convenient to remove the skew by shearing the image along the range time axis by the amount

$$
\delta_{\tau}=-\left(\frac{V_{\mathrm{T}} \sin \left(\theta_{\mathrm{T}}\right)+V_{\mathrm{R}} \sin \left(\theta_{\mathrm{R}}\right)}{c}\right) \eta .
$$

The deskewed sidelobes are seen in Fig. 2(d). The deskewing operation is equivalent to deskewing the spectrum, as shown in Fig. 2(c).

The quality of the focus can be examined using the onedimensional expansions shown in Fig. 3. The excellent focus is demonstrated by the IRW, which meets the theoretical limits 
(a)

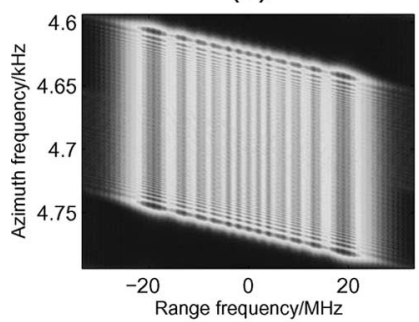

(c)

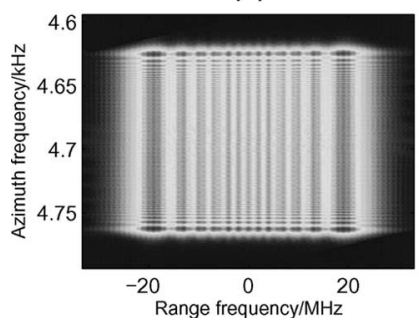

(b)

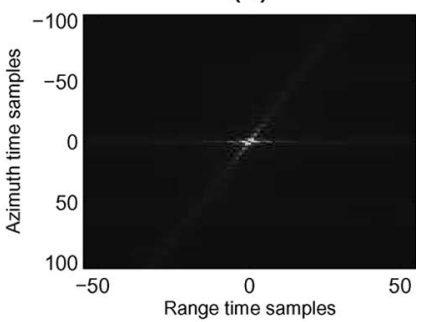

(d)

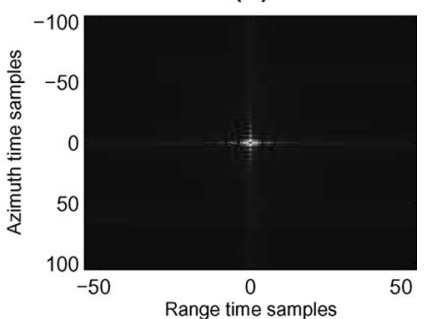

Fig. 2. Point target spectrum and image before and after the shear operation (a) Spectrum after matched filtering. (b) Point target after matched filtering. (c) Spectrum after shear operation. (d) Point target after shear operation.
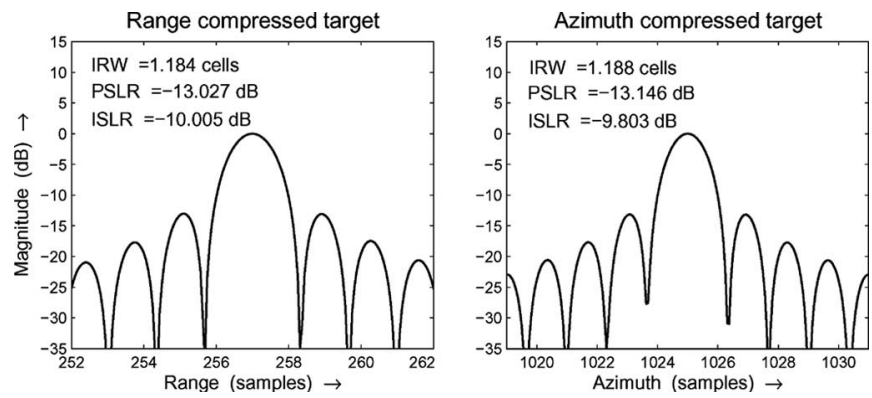

Fig. 3. Measurement of point target focus using a matched filter derived from the new 2-D point target spectrum.

in range $(1.184 / 1.33=0.89)$ and in azimuth $(1.188 / 1.33=$ $0.89)$ for rectangular weighting. Furthermore, the sidelobes agree with the theoretical values of -10 and $-13.3 \mathrm{~dB}$ for the integrated sidelobe ratio (ISLR) and PSLR, respectively. In addition, the symmetry of the sidelobes is another indication of correct matched filter phase.

\section{CONClusion}

The 2-D point target spectrum for the general bistatic case is developed by expressing the bistatic range equation as a power series and using the method of series reversion to express azimuth time as a function of azimuth frequency during the azimuth FT. This results in a power series expression for the spectrum of the point target, whose accuracy is controlled by the degree of the power series. The accuracy of the derived spectrum is confirmed using a simulation where the point target is simulated in the time domain, then compressed using a 2-D matched filter derived from the spectrum.

The method of series reversion is also applicable to monostatic stripmap and spotlight situations where the simple hyperbolic range equation does not hold.

\section{APPENDIX \\ SERIES REVERSION}

Series reversion is the computation of the coefficients of the inverse function given those of the forward function (26). For a function expressed in a series with no constant term $a_{0}=0$

$$
y=a_{1} x+a_{2} x^{2}+a_{3} x^{3}+\cdots
$$

the series expansion of the inverse function is given by

$$
x=A_{1} y+A_{2} y^{2}+A_{3} y^{3}+\cdots .
$$

Substituting (27) into (26), the following equation is obtained:

$$
\begin{aligned}
y=a_{1} A_{1} y+ & \left(a_{2} A_{1}^{2}+a_{1} A_{2}\right) y^{2} \\
& +\left(a_{3} A_{1}^{3}+2 a_{1} A_{1} A_{2}+a_{1} A_{3}\right) y^{3}+\cdots .
\end{aligned}
$$

By equating terms, the coefficients of the inverse function are

$$
\begin{aligned}
A_{1} & =a_{1}^{-1} \\
A_{2} & =-a_{1}^{-3} a_{2} \\
A_{3} & =a_{1}^{-5}\left(2 a_{2}^{2}-a_{1} a_{3}\right) \\
& \ldots
\end{aligned}
$$

The formula for the $n$th coefficient is given in [10], as summarized in the handbook [11].

\section{ACKNOWLEDGMENT}

The authors would like to thank DSO National Laboratories, Singapore, for providing scholarship funding for Y. L. Neo.

\section{REFERENCES}

[1] B. Barber, "Theory of digital imaging from orbital synthetic aperture radar," Int. J. Remote Sens., vol. 6, no. 6, pp. 1009-1057, 1985.

[2] I. G. Cumming and F. H. Wong, Digital Processing of Synthetic Aperture Radar Data: Algorithms and Implementation. Norwood, MA: Artech House, 2005.

[3] R. K. Raney, "A new and fundamental Fourier transform pair," in Proc. Int. Geosci. Remote Sens. Symp., Clear Lake, TX, May 1992, pp. 106-107.

[4] O. Loffeld, H. Nies, V. Peters, and S. Knedlik, "Models and useful relations for bistatic SAR processing," IEEE Trans. Geosci. Remote Sens., vol. 42, no. 10, pp. 2031-2038, Oct. 2004.

[5] D. D'Aria, M. Guarnieri, and F. Rocca, "Focusing bistatic synthetic aperture radar using dip move out," IEEE Trans. Geosci. Remote Sens., vol. 42, no. 7, pp. 1362-1376, Jul. 2004.

[6] P. Dubois-Fernandez, H. Cantalloube, B. Vaizan, G. Krieger, R. Horn, M. Wendler, and V. Giroux, "ONERA-DLR bistatic SAR campaign: Planning, data acquistion, and first analysis of bistatic scattering behaviour of natural and urban targets," Proc. Inst. Electr. Eng.-Radar, Sonar Navig., vol. 153, no. 3, pp. 214-223, Jun. 2006.

[7] I. Walterscheid, A. Brenner, and J. Ender, "Geometry and system aspects for a bistatic airborne SAR-experiment," in Proc. EUSAR, Ulm, Germany, May 2004, pp. 567-570.

[8] Y. Neo, F. Wong, and I. G. Cumming, "An efficient non-linear chirp scaling method of focusing bistatic SAR images," in Proc. EUSAR, Dresden, Germany, May 2006. [CD-ROM].

[9] A. Papoulis, Signal Analysis. New York: McGraw-Hill, 1977.

[10] P. M. Morse and H. Feshbach, Methods of Theoretical Physics, 1st ed. New York: McGraw-Hill, 1953.

[11] H. B. Dwight, Table of Integrals and Other Mathematical Data, 4th ed. New York: Macmillan, 1961. 